The etiology of this affection is somewhat obscure. The patients are usually of delicate constitution, and may or may not have had previous catarrh or irritation of the urethra, perhaps from oxyuria. Masturbation should be taken into account. Persistent cough, as during pertussis, is a possible etiological factor.

In one case, in which the prolapse was less marked, the patient was cured by an application of the cautery. In another an equally favorable result was obtained by replacing the prolapsed mucosa, and narrowing the urethra temporarily with sutures until the redundant tissue was reduced by repeated cauterization. If this does not succeed, the simplest plan is to excise the prolapsed mass and to suture the raw edges with catgut.

\title{
The Electrical Treatment of Cancer of the Uterus.
}

Wernitz (Berliner klin. Wochenschrift, September 22, 1890) limits this treatment to cases that are nearly, or quite, inoperable. A large, flat electrode being placed over the abdomen, and a ball electrode applied through a speculum directly to the discased surface, a current varying from one to two hundred milliampères is passed for not longer than ten minutes; a séance is held daily or every other day. In some cases the growth is punctured with a platinum needle attached to the negative pole. When the positive pole is used a thin grayish slough appears at the point of contact, which separates within twenty-four hours, leaving a clean raw surface, that has less tendency to bleed; with the negative pole there is no visible slough, but a secretion of fluid-

Four cases are reported at length, in which the electrical treatment caused entire cessation of the pain, so that opium could be dispensed with, and marked improvement in the appetite and general condition. Sloughing and foul discharge ceased. The action of the current in these cases is probably two-fold, a local or chemical, whereby healthy granulation and cicatrization are promoted, and an electrolytic, in consequence of which there is a molecular disintegration and absorption of the cancer-cells in the deeper tissues.

\section{P丑DIATRIOS.}

UNDER THE CHARGE OF

JOHN M. KEATING, M.D.,

or PHILAdEliphia,
A. F. CURRIER, M.D.,
AND
W. A. EDW ARDS, M.D.,
OF SAN DiEgo, CAL.

The Buccal Phenomena and Complications of Measles in Children.

Cauvet (Gaz. Méd. de Paris, May 31, 1890) claims that during measles there may be two varieties of lesions of the mouth, one being due to the infection of the disease, the other being a superadded complication. The phenomena due to the infection of measles are of two kinds: 1st. Buccal erythema, 
which is usually present at the same time as the nasal catarrh, and consists in a red spottiness, or a simple redness upon the tongue. It may be followed by desquamation of the tongue. 2d. Follicular stomatitis, due to glandular hyper-secretion with obstruction of the excretory canal, and coinciding ordinarily with the miliary eruption of the skin. These phenomena may be due to the poison of the disease, which is eliminated by the glands. The oral complications which are superadded to measles may vary in nature and gravity, and may show the following forms:

1. Muguet, which is the most frequent complication.

2. Aphthæ upon the tongue, the lips, or the internal aspect of the cheek.

3. Ulcerative stomatitis, which may follow rupture of the aphthæ, or may result from infection which has been superadded.

4. Diphtheria, which, as has been shown by Roux and Yersin, may develop whenever there is epithelial desquamation. There is frequently a lingual desquamation following buccal erythema. In other conditions, aside from measles, diphtheria is unlikely first to attack the tongue.

5. Gangrene of the mouth, which is a serere though rare complication.

To avoid these accidents antiseptic washes should be freely used for the buccal cavity. A saturated solution of boric acid will be found very serviceable for this purpose. The washes should be used from the very beginning of the disease, when there may be only a mild degree of erythematous stomatitis. These complications are much more common in hospitals than in private practice, and this is fortunate, for in hospitals they are much more likely to receive the attention that is required.

\section{Croupous Pneumonia in Children and its Treatment.}

Copasso (Arch. Ital. di Ped., 1890) states that between the first week and the sixth year of life children are very susceptible to this disease. Those are especially predisposed to the disease in whom the thorax is undeveloped relatively to other parts of the body. Cutaneous erythema upon the trunk is one of the first symptoms, especially in severe cases, and is probably due to obstruction to the flow of blood toward the thoracic cavity. To the vascular obstruction are also due the albuminuria of the disease, the congestion of the liver, the spleen, and the brain, the softness of the tissues offering no opposition to the dilatation of the capillaries. In cerebral pneumonia the morbid process is usually limited to the left apex, while there is also cdema of the pia mater. The posterior portion of the lung is more decidedly affected than the lateral or inferior portions. Cardiac weakness may follow the disease, and the pulmonary vesicles very slowly resume their original form and volume. Caseation and tuberculization of the exudate in such young patients are rare, and so also are induration, suppuration, and gangrene. It is believed by the author that the theory of the infectious origin of the disease is a correct one. Salicine may not be given with safety at this period of life. Leeches may be used with advantage to the painful portions of the chest. Excitants of the nervous system and the heart, including red wine and quinine, also expectorants, may be prescribed. 